\title{
Vulnerabilidade domiciliar em situação de intermitência no abastecimento de água
}

\author{
Household vulnerability to intermittent water supply systems
}

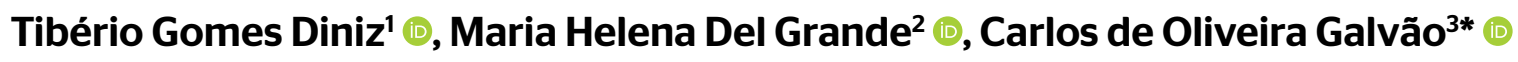

口

\section{RESUMO}

Os sistemas de abastecimento em regime de intermitência fornecem água aos usuários por um período limitado de tempo, produzindo efeitos adversos à rede de abastecimento de água e aos usuários do sistema, em especial os usuários domésticos, cuja demanda é pouco flexível e a capacidade de adaptação limitada. Neste trabalho é proposto um conjunto simplificado de indicadores que permite identificar diferentes níveis de vulnerabilidade ao desabastecimento de água aos quais uma população urbana está sujeita em situação de intermitência no abastecimento. Os indicadores podem ser obtidos de dados censitários, de informações topológicas, topográficas e hidráulicas do sistema de abastecimento e de levantamentos cadastrais ou de sensoriamento remoto da localidade, podendo ser processados em Sistemas de Informações Geográficas (SIGs). Os indicadores são aplicados ao caso de Campina Grande, Paraíba, com base em levantamentos disponíveis sobre usuários que vivenciaram a situação de intermitência no abastecimento. Os resultados permitem a identificação das áreas cujos usuários podem ser potencialmente mais afetados pela intermitência no abastecimento e a recomendação de medidas mitigadoras para a redução de sua vulnerabilidade.

Palavras-chave: indicadores de vulnerabilidade; Campina Grande; escassez.

\begin{abstract}
Intermittent supply in water distribution systems occurs when the water service is available for a limited period of time, producing adverse effects on the water supply network and users of the system, in particular domestic users, whose demand is not flexible and the adaptive capacity is limited. This paper proposed a simplified set of indicators to identify different levels of vulnerability to water shortages to which an urban population is subject, in a situation of intermittent water supply. The indicators can be obtained from census data, topological, topographic, and hydraulic information from the supply system and from cadastral surveys or remote sensing of the locality, and can be processed in geographic information systems (GIS). This article presented the formulation of the indicators and their application to the Campina Grande system, Paraíba, Brazil, based on available surveys on users in intermittent supply situations. The results allow the identification of areas whose users may be potentially most affected by intermittent supply and the recommendation of mitigating measures to reduce their vulnerability.
\end{abstract}

Keywords: vulnerability indicators; Campina Grande; scarcity.

\section{INTRODUÇÃO}

Os sistemas de abastecimento de água são projetados para atender à população de forma ininterrupta, porém têm sofrido com a ocorrência mais frequente do funcionamento intermitente (ILAYA-AYZA et al., 2017). A intermitência ocorre quando o serviço de abastecimento de água fica disponível aos usuários menos de 24 horas por dia. Essa é uma condição indesejável, ocasionada por problemas estruturais e/ou operacionais ou por situações de escassez de água, tais como a redução da disponibilidade hídrica provocada pela variabilidade climática e pela degradação das bacias hidrográficas, a crescente demanda decorrente do crescimento populacional e as dinâmicas econômicas locais, regionais e nacionais. A intermitência produz efeitos adversos, como deterioração da rede de distribuição de água, normalmente projetada para abastecimento contínuo, perdas de água na forma de vazamentos, ajustes constantes no balanço hídrico do sistema de abastecimento e necessidade de adaptações dos usuários, em especial os domésticos, cuja demanda é pouco flexível e a capacidade de armazenamento de

${ }^{1}$ Centro Universitário de Patos - Patos (PB), Brasil.

${ }^{2}$ Instituto Federal de São Paulo - São Paulo (SP), Brasil.

${ }^{3}$ Universidade Federal de Campina Grande - Campina Grande (PB), Brasil.

*Autor correspondente: carlos.o.galvao@gmail.com

Conflitos de interesse: os autores declaram não haver conflito de interesses.

Financiamento: Conselho Nacional de Desenvolvimento Científico e Tecnológico, Coordenação de Aperfeiçoamento de Pessoal de Nível Superior, Agência Nacional de Águas, Financiadora de Estudos e Projetos e Fundo Setorial de Recursos Hídricos, do Ministério da Ciência, Tecnologia e Inovações

Recebido: 10/O2/2019 - Aceito: 08/06/2O2O - Reg. ABES: 20190038 
água interna ao domicílio é limitada, entre outros (AGATHOKLEOUS; CHRISTODOULOU, 2016; SIMUKONDA; FARMANI; BUTLER, 2018; BORUFF et al., 2018).

As análises de vulnerabilidade dos usuários domésticos a tais situações indesejáveis são importantes para identificação das zonas do sistema que necessitam de ajustes, para redução dos prejuízos aos usuários lá localizados. São frequentemente realizadas agregando indicadores que representam os fatores associados aos impactos. Ao agregar esses indicadores é possível comparar diferentes graus de vulnerabilidade (CUTTER; BORUFF; SHIRLEY, 2003; RUNFOLA et al., 2017). Na agregação podem ser utilizadas técnicas multicriteriais e Sistema de Informações Geográficas (SIG). Como exemplo, Malta, Costa e Magrini (2017) elaboraram um índice representativo de variáveis sociais, econômicas, de infraestrutura urbana, ambientais, de saúde e de segurança pública na cidade do Rio de Janeiro, Rio de Janeiro, por meio da sobreposição de indicadores e espacialização dos resultados. Grande et al. (2014) propuseram um modelo de análise da vulnerabilidade ao desabastecimento urbano de água baseado na agregação dos critérios: econômico - renda mensal familiar média por setor censitário; social - presença de aglomerados subnormais; hidráulico — risco de desabastecimento; e mitigador - a presença de reservatórios de água em condomínios verticais; considerando períodos de escassez hídrica nos quais o racionamento de água foi aplicado.

Este artigo propõe um conjunto simplificado de indicadores para análise da vulnerabilidade ao desabastecimento de água em situações de intermitência no abastecimento e medidas de intervenções para atenuação dessa vulnerabilidade, tendo como base o trabalho de Grande et al. (2014), usando dados disponíveis e/ou facilmente adquiridos, que possam indicar diferentes níveis de vulnerabilidade de qualquer cidade e subsidiar a apresentação de medidas mitigadoras por meio da localização das áreas propícias de intervenção no risco hidráulico. O conjunto de indicadores foi aplicado ao caso de Campina Grande, Paraíba, cidade de médio porte, de clima semiárido, cuja população convive com a intermitência no abastecimento de água, o que gera distribuição desigual de água para os usuários e indica situação de escassez hídrica estrutural (GRANDE, 2016).

\section{METODOLOGIA}

\section{Caso de estudo}

O objeto de estudo foi a área urbana da cidade de Campina Grande, Paraíba, localizada na região semiárida do Nordeste brasileiro (Figura 1). A população residente nos $96 \mathrm{~km}^{2} \mathrm{da}$ área urbana é de 385.213 habitantes (IBGE, 2010), correspondente a $95 \%$ da população total do município. Segundo o último Censo Demográfico, a renda per capita média de Campina Grande era de R \$ 630,03, a porcentagem de pobres era de $16,34 \%$ e o Índice de Gini, utilizado para medir o grau de concentração de renda e que varia entre 0 e 1 , era de 0,58 , caracterizando situação de desigualdade de renda (IBGE, 2010).

Campina Grande e mais 26 municípios menores são abastecidos pelo Reservatório Epitácio Pessoa, localizado a cerca de $45 \mathrm{~km}$, no município de Boqueirão. Toda a bacia hidrográfica que contribui para esse reservatório está situada na região mais seca do país, caracterizada por regime irregular de precipitação que, em longos períodos de estiagem, enfrenta severas crises hídricas. Essas condições climáticas, associadas ao alto consumo e às perdas $\mathrm{e}$ deficiências de gestão das águas, levaram o Reservatório Epitácio Pessoa a duas grandes crises hídricas, a primeira, de 1998 a 2000, e a mais recente, de 2012 a 2017, as quais produziram impactos no abastecimento de água de Campina Grande, dentre eles o agravamento da intermitência no abastecimento (RÊGO et al., 2017). Em ambos os episódios, a medida de gestão tomada foi o racionamento de água, que agravou consideravelmente a vulnerabilidade das populações mais expostas e sensíveis aos efeitos negativos do desabastecimento de água (GRANDE et al., 2016; RÊGO et al., 2017).

Segundo Grande (2016), a distribuição de água na área urbana de Campina Grande é desigual, evidenciada por dificuldades no funcionamento pleno e regular do sistema de abastecimento, decorrentes do relevo acidentado (altitudes que variam de 337 a $665 \mathrm{~m}$ acima do nível do mar), de alterações e ampliações na rede de distribuição de água não acompanhadas por soluções de engenharia para equacionar oferta e demanda e de uma gestão ineficiente dos recursos hídricos, indicando situação de escassez hídrica estrutural. Grande (2016) selecionou uma amostra intencional de 23 domicílios, todos localizados na área urbana do município de Campina Grande, e, a partir dela, identificou, durante o período do último racionamento, as populações em áreas com deficiências operacionais da rede de distribuição que estiveram mais vulneráveis. Observou que as de maior renda domiciliar tinham maior capacidade de reservação no domicílio e maior facilidade no acesso à água, enquanto as de menor renda domiciliar tinham menor capacidade de reservação no domicílio e/ou dispendiam maior esforço para acesso à água.

\section{Unidade de análise}

A disponibilidade de dados censitários determinou a escolha do setor censitário como unidade de análise, que compreende, em regiões urbanas, cerca de 300 domicílios (IBGE, 2010). Apesar de ainda ser uma escala pouco adequada para análises detalhadas da vulnerabilidade domiciliar, a escala do setor censitário apresenta vantagens por permitir captar as diversidades sociodemográfica e ambiental na cidade (ALVES, 2000).

\section{Seleção do conjunto de indicadores de vulnerabilidade e aquisição dos dados}

No presente estudo, foi adotado o conceito de Mukesh, Komal e Alexander (2017), segundo o qual a vulnerabilidade é função de três dimensões: a exposição, caracterizada pela susceptibilidade física ao desastre; a sensibilidade, representada pela fragilidade socioeconômica; e a capacidade de adaptação, que é caracterizada pelas respostas aos efeitos do desastre. A escolha pelos indicadores de vulnerabilidade foi baseada nos estudos de Grande et al. (2014) e de Grande (2016), considerando-se os critérios econômico, social, hidráulico e de capacidade adaptativa, em períodos de abastecimento regular com ocorrências de intermitência: risco hidráulico - dimensão exposição física; renda mensal média domiciliar - dimensão sensibilidade econômica; presença de aglomerados subnormais no setor censitário - dimensão sensibilidade social; e capacidade de reservação - dimensão capacidade adaptativa. O Quadro 1 apresenta os indicadores selecionados e as respectivas informações referentes às dimensões da vulnerabilidade as quais eles representam, a justificativa para escolha de cada indicador, as classes utilizadas e a forma de aquisição dos dados.

Os dados de renda mensal domiciliar por setor censitário foram obtidos a partir da divisão dos valores da variável V003 (“Total do rendimento nominal 


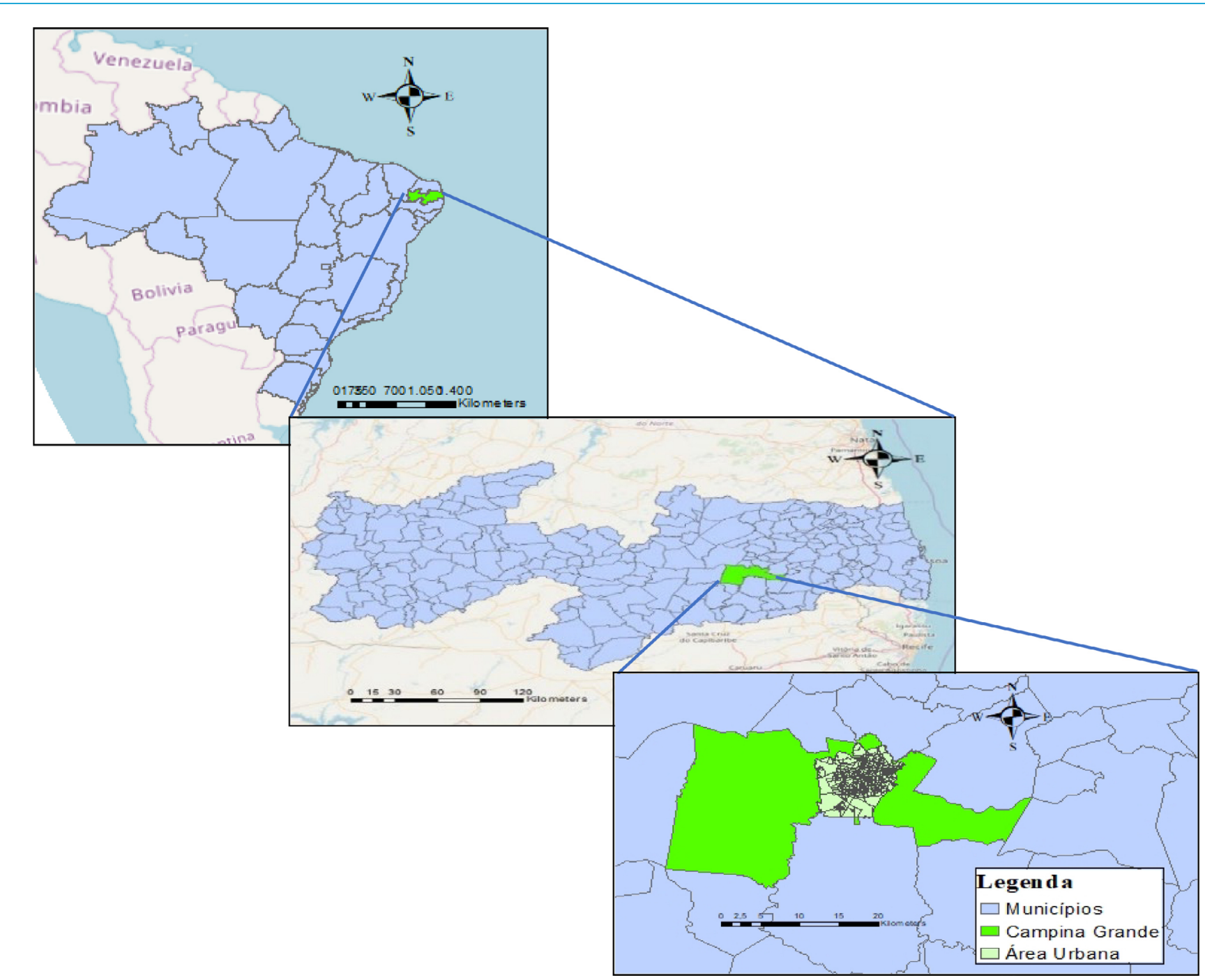

Fonte: com base em SEPLAN e PMCG (2014).

Figura 1 - Delimitação da área de estudo.

mensal dos domicílios particulares permanentes", Planilha DomicílioRenda_ UF.xls) pela variável V002 ("Domicílios particulares permanentes", Planilha Domicilio01_PB.xls) do Censo 2010 (IBGE, 2010). Os dados sobre a presença de aglomerados subnormais nos setores censitários foram obtidos a partir da variável Tipo_setor, da Planilha Básico_UF.xls (IBGE, 2010).

Para aquisição dos dados do indicador Capacidade de Reservação, foi utilizado um mosaico de imagens aéreas de alta resolução espacial $(10 \times 10 \mathrm{~cm})$ e sua respectiva representação vetorial em forma de mapa digital com informações de delimitações do lote, uso do solo e de edificações do meio urbano (Figura 2A). Essas informações foram cedidas pela Secretaria de Planejamento (SEPLAN) da Prefeitura Municipal de Campina Grande (PMCG). A identificação dos reservatórios e sua contagem ocorreram por meio da interpretação visual da imagem, utilizando-se um SIG. Foram considerados como reservatórios: caixas d'água de polietileno (Figura $2 \mathrm{~B}$ ), caracterizadas por um polígono circular azul no telhado dos domicílios; caixas d'água de concreto/ alvenaria/fibrocimento (Figura $2 \mathrm{C}$ ), caracterizadas por polígonos retangulares de cor escura diferente da cor do telhado; e piscinas (Figura 2D), levando-se em consideração que as piscinas constituem também uma forma de armazenamento de água, podendo ser detectadas a partir de um polígono circular, elipsoidal ou retangular azul nos domicílios. Dessa forma, para saber a quantidade total de domicílios com reservatório e o seu percentual por setor censitário, considerando os condomínios verticais, foram utilizadas as variáveis V005 e V002 da planilha Domicilio01_PB.xls (IBGE, 2010), respectivamente a quantidade de domicílios particulares permanentes do tipo apartamento por setor censitário e a quantidade de domicílios particulares permanentes. Assumindo-se que os domicílios tipo apartamento têm caixas d'água, a quantidade total de domicílios com reservatório é obtida por meio da soma da contagem dos domicílios com reservatório com os domicílios do tipo apartamento (V005). Vale destacar que os dados referentes a esse indicador têm certo grau de incerteza, o qual não foi avaliado neste estudo, devido à possível presença de outros reservatórios nos domicílios, não detectáveis na interpretação visual da imagem. 
Quadro 1 - Indicadores de vulnerabilidade em abastecimento intermitente.

\begin{tabular}{|c|c|c|c|c|}
\hline Indicador & $\begin{array}{l}\text { Dimensão da } \\
\text { vulnerabilidade }\end{array}$ & Justificativa & Classes & Aquisição dos dados \\
\hline Risco hidráulico & Exposição física & $\begin{array}{l}\text { Quanto maior a elevação e a distância do } \\
\text { domicílio em relação ao reservatório de } \\
\text { distribuição, maior o risco de desabastecimento } \\
\text { no domicílio e maior a vulnerabilidade }\end{array}$ & $\begin{array}{l}\text { - Alto } \\
\text { - Médio } \\
\text { - Baixo }\end{array}$ & $\begin{array}{l}\text { Mapa de risco de desabastecimento em } \\
\text { Campina Grande elaborado por Cordão, } \\
\text { Rufino e Araújo (2013) }\end{array}$ \\
\hline $\begin{array}{l}\text { Renda mensal } \\
\text { domiciliar }\end{array}$ & $\begin{array}{l}\text { Sensibilidade } \\
\text { econômica }\end{array}$ & $\begin{array}{l}\text { Os domicílios de baixa renda são mais } \\
\text { vulneráveis do que os domicílios de média e } \\
\text { alta rendas }\end{array}$ & $\begin{array}{l}\text { - Até } 3 \text { salários mínimos } \\
\text { - De } 3 \text { a } 5 \text { salários mínimos } \\
\text { - Acima de } 5 \text { salários mínimos }\end{array}$ & $\begin{array}{l}\text { Variáveis do Censo 2010: } \\
\text { - VOO3 ("Total do rendimento nominal mensal } \\
\text { dos domicílios particulares permanentes", } \\
\text { Planilha DomicílioRenda_UF.xIS) } \\
\text {-VOO2 (“Domicílios particulares permanentes", } \\
\text { Planilha DomicilioO__PB.xIs) } \\
\text { (IBGE, 2O10). }\end{array}$ \\
\hline $\begin{array}{l}\text { Presença de } \\
\text { aglomerados } \\
\text { subnormais no } \\
\text { setor censitário }\end{array}$ & $\begin{array}{l}\text { Sensibilidade } \\
\text { social }\end{array}$ & $\begin{array}{l}\text { Os domicílios localizados em assentamentos } \\
\text { irregulares (favelas, invasões, etc) apresentam } \\
\text { maior vulnerabilidade socioambiental (MALTA; } \\
\text { COSTA; MAGRINI, 2017) }\end{array}$ & $\begin{array}{l}\text { - Aglomerados subnormais } \\
\text { - Outros }\end{array}$ & IBGE (2010) \\
\hline $\begin{array}{l}\text { Capacidade de } \\
\text { reservação }\end{array}$ & $\begin{array}{l}\text { Capacidade } \\
\text { adaptativa }\end{array}$ & $\begin{array}{l}\text { A presença de reservatórios no domicílio } \\
\text { aumenta a continuidade do acesso à água, } \\
\text { mesmo em casos de intermitência no } \\
\text { abastecimento, portanto este indicador } \\
\text { é considerado adaptativo em relação à } \\
\text { vulnerabilidade (GRANDE, 2016) }\end{array}$ & $\begin{array}{l}\text { Percentual dos domicílios } \\
\text { com capacidade de } \\
\text { reservação por setor } \\
\text { censitário: } \\
\text { - Abaixo de } 50 \% \\
\text { - Acima de } 50 \%\end{array}$ & $\begin{array}{l}\text { Mosaico de imagens aéreas de alta resolução } \\
\text { espacial }(10 \times 10 \text { cm) de Campina Grande } \\
\text { (SEPLAN; PMCG, 2014) }\end{array}$ \\
\hline
\end{tabular}

Fonte: elaborado pelos autores.

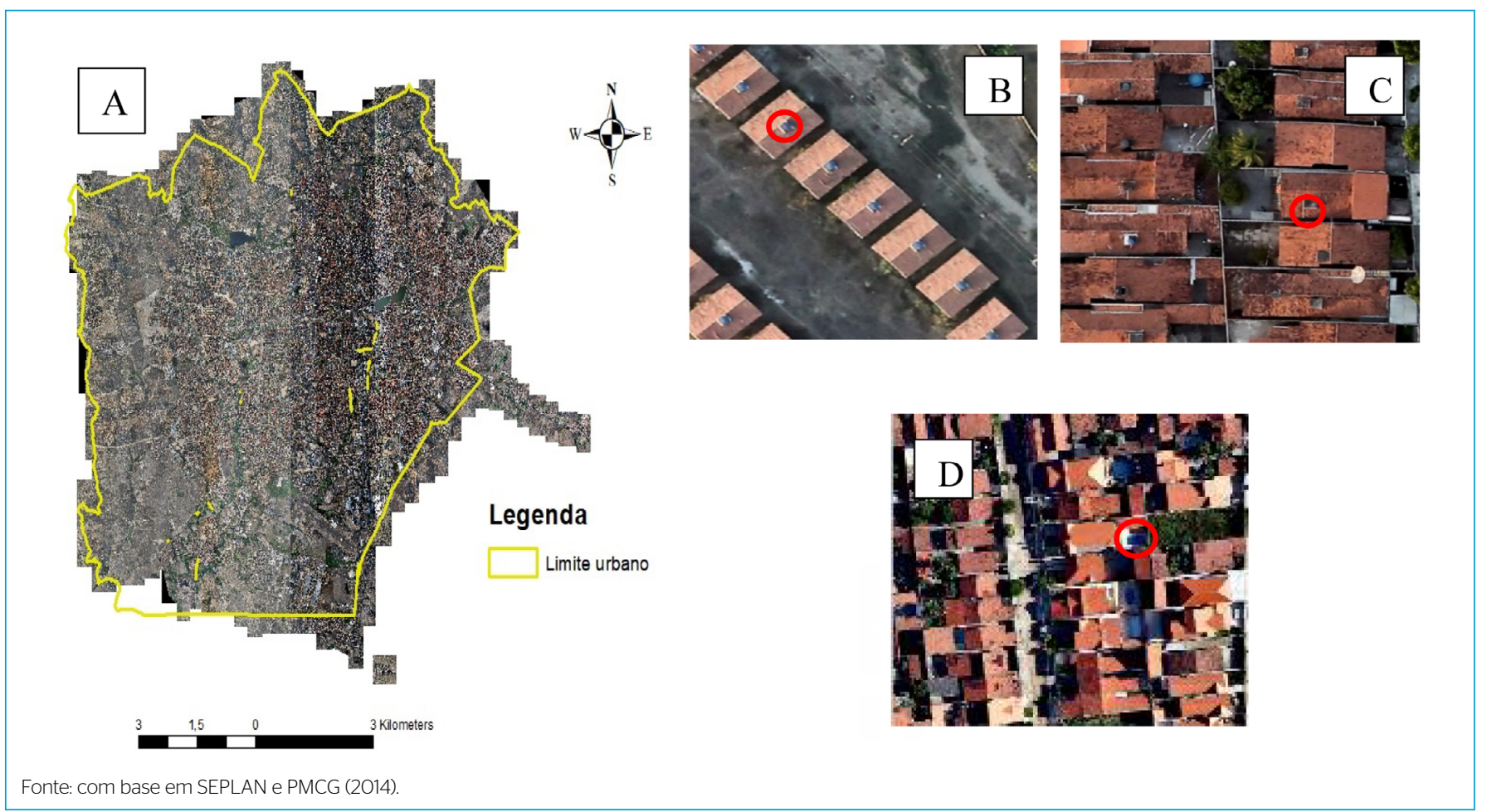

Figura 2 - (A) Mosaico de imagem com representação vetorial de Campina Grande; (B) caixas d’água de polietileno; (C) caixas d’água de concreto/alvenaria/fibrocimento; (D) piscinas.

\section{Tratamento dos dados em SIG}

Assim como em estudos anteriores, como o de Grande et al. (2014), empregou-se uma ferramenta SIG, a partir do programa ArcGIS 10.5, para agregar os indicadores de vulnerabilidade selecionados: risco hidráulico, renda mensal domiciliar e presença de aglomerados subnormais, por meio das regras de decisão apresentadas no Quadro 2. A vulnerabilidade foi classificada em: muito alta, alta, média, baixa e muito baixa.

Foram elaborados mapas temáticos para cada indicador e, a partir deles, utilizando-se álgebra de mapas, considerando-se as regras de decisão estabelecidas no Quadro 2, foi gerado o mapa de vulnerabilidade. A reservação 
Quadro 2 - Regras de decisão estabelecidas para agregação dos indicadores risco hidráulico, renda mensal domiciliar e presença de aglomerados subnormais.

\begin{tabular}{|c|c|c|c|c|}
\hline \multirow{2}{*}{\multicolumn{2}{|c|}{ Vulnerabilidade }} & \multicolumn{3}{|c|}{$\begin{array}{l}\text { Renda mensal domiciliar e aglomerados } \\
\text { subnormais }\end{array}$} \\
\hline & & $\begin{array}{c}\text { Até } 3 \text { salários mínimos } \\
\text { OU aglomerado } \\
\text { subnormal }\end{array}$ & $\begin{array}{l}\text { De } 3 \text { a } 5 \\
\text { salários } \\
\text { mínimos }\end{array}$ & $\begin{array}{l}\text { Acima de } 5 \\
\text { salários } \\
\text { mínimos }\end{array}$ \\
\hline \multirow{3}{*}{$\begin{array}{l}\text { Risco } \\
\text { hidráulico }\end{array}$} & Alto & Muito alta & Alta & Média \\
\hline & Médio & Alta & Média & Baixa \\
\hline & Baixo & Média & Baixa & Muito baixa \\
\hline
\end{tabular}

Fonte: elaborado pelos autores.

no domicílio tem efeito mitigador sobre a vulnerabilidade, por se tratar de capacidade adaptativa diante da condição de intermitência no abastecimento de água. Apesar de contribuir para a redução da vulnerabilidade do usuário, a capacidade de reservação não melhora a condição de desabastecimento de água em si, pois se trata de uma medida particular, adotada pelo usuário para solução do seu problema, sem atuar ou modificar a sua causa. O indicador capacidade de reservação foi agregado ao modelo conforme as regras de decisão apresentadas no Quadro 3, sendo gerado o mapa de vulnerabilidade atenuada.

A vulnerabilidade de um setor censitário com mais que $50 \%$ dos seus domicílios com capacidade de reservação é, então, reduzida para a classe inferior, com exceção do caso em que a vulnerabilidade é muito baixa e do caso em que a vulnerabilidade é alta: segundo os dados obtidos a partir da amostra intencional da pesquisa conduzida por Grande (2016), a capacidade de reservação está associada à renda e requer maior esforço físico dos usuários de menor renda para ter acesso à água reservada, sendo que a predominância de domicílios em áreas de vulnerabilidade alta é de rendas baixa e média, o que levou a manter a classe de vulnerabilidade atenuada alta nesses casos.

\section{RESULTADOS E DISCUSSÃO}

Com base nos mapas dos indicadores risco hidráulico (Figura 3), renda média mensal por setor censitário (Figura 4) e aglomerados subnormais (Figura 5), os dados foram sobrepostos, gerando o mapa de vulnerabilidade de acordo com as regras de decisão estabelecidas no Quadro 2 (Figura 6). A operação do mapa de vulnerabilidade da Figura 6 com o mapa do indicador capacidade de reservação (Figura 7), por meio das regras de decisão estabelecidas no Quadro 3, gerou o mapa de vulnerabilidade atenuada (Figura 8).

O risco hidráulico (Figura 3) é maior nas áreas mais distantes da região central da cidade, resultado da expansão recente e elevada da área urbana, não acompanhada satisfatoriamente pela rede de abastecimento, com exceção das áreas periféricas de topografia mais baixa. É possível observar nas Figuras $3 \mathrm{e}$ 6 que o indicador risco hidráulico influencia na vulnerabilidade ao desabastecimento de água em situações de intermitência no abastecimento, delimitando as regiões com vulnerabilidade muito alta pelo alto risco hidráulico. As áreas delimitadas pelo risco hidráulico (Figura 3) se mantiveram, em sua maioria, no mapa de vulnerabilidade da Figura 6, mesmo após agregação dos outros indicadores selecionados.
Quadro 3 - Regras de decisão estabelecidas para agregação do indicador capacidade de reservação.

\begin{tabular}{|c|c|c|}
\multirow{4}{*}{ Vulnerabilidade atenuada } & $\begin{array}{c}\text { Percentual de domicilios com capacidade } \\
\text { de reservação }\end{array}$ \\
\cline { 2 - 3 } Vulnerabilidade & Muito alta & Acima de $50 \%$ \\
\cline { 2 - 3 } & Alta & Alta \\
\cline { 2 - 3 } & Média & Alta \\
\cline { 2 - 3 } & Baixa & Baixa \\
\hline & Muito baixa & Muito baixa \\
\hline
\end{tabular}

Fonte: elaborado pelos autores.

Nos mapas das Figuras 4 e 5 e no mapa de vulnerabilidade da Figura 6 é possível detectar que em algumas regiões a presença de aglomerados subnormais e a renda média mensal baixa contribuíram para gerar um grau de vulnerabilidade relativamente maior. Embora delimitadas pelo risco hidráulico, essas regiões tiveram sua vulnerabilidade ao desabastecimento de água compensada por suas condições socioeconômicas, pois se trata de áreas com risco hidráulico médio e baixo, porém com renda média até no máximo 3 salários mínimos ou com presença de aglomerados subnormais, gerando vulnerabilidade média e alta, respectivamente, mas não chegando a ter vulnerabilidade muito alta.

Pela Figura 7 pode-se afirmar que na área urbana de Campina Grande poucos setores censitários apresentam mais que $50 \%$ de seus domicílios com capacidade de reservação, podendo-se concluir que a capacidade adaptativa do município é baixa. Por outro lado, mesmo que ainda sejam poucos os setores censitários com boa capacidade adaptativa, ao agregar essas informações ao mapa de vulnerabilidade gerado pelo conjunto de indicadores (Figura 6) para avaliar a vulnerabilidade, sua vulnerabilidade foi reduzida (Figura 8).

Os mapas de vulnerabilidade e vulnerabilidade atenuada foram comparados aos mapas de vulnerabilidade e vulnerabilidade final, elaborados por Grande et al. (2014), sendo possível observar que o aumento do número de classes de vulnerabilidade, bem como o refinamento dos dados referentes à capacidade de reservação, forneceu informações mais detalhadas sobre a vulnerabilidade ao desabastecimento, com destaque para a vulnerabilidade média, não detectada no estudo anterior. Enquanto os setores censitários sem vulnerabilidade do estudo anterior coincidem, em grande parte, com os setores com vulnerabilidades baixa e muito baixa deste estudo, os setores com vulnerabilidade muito alta e alta do estudo anterior também coincidem com os setores com vulnerabilidade muito alta deste estudo, e os setores com Vulnerabilidade alta e média mostraram diferenciação não percebida no estudo anterior, conforme se discutirá a seguir.

Para essa comparação, os mapas de vulnerabilidade e vulnerabilidade atenuada deste estudo foram comparados aos de vulnerabilidade e vulnerabilidade final elaborados por Grande et al. (2014). Os resultados de vulnerabilidade foram associados aos dados dos domicílios do caso de estudo de Grande (2016), conforme Tabela 1, observando-se a influência dos indicadores, especialmente o risco hidráulico e a capacidade de reservação. Para os 23 domicílios da amostra intencional de Grande (2016), 11 deles (48\%) apresentaram a mesma classe de vulnerabilidade neste estudo e no de Grande et al. (2014). 


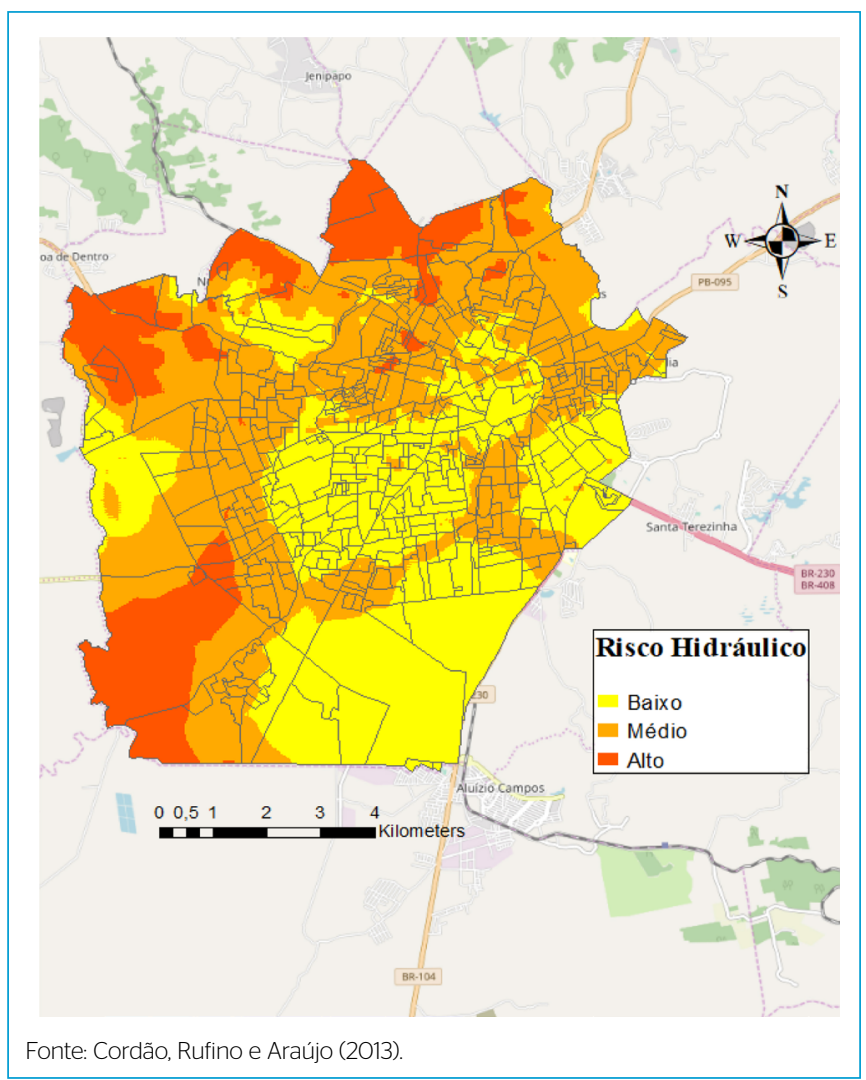

Figura 3 - Risco hidráulico na área urbana de Campina Grande.

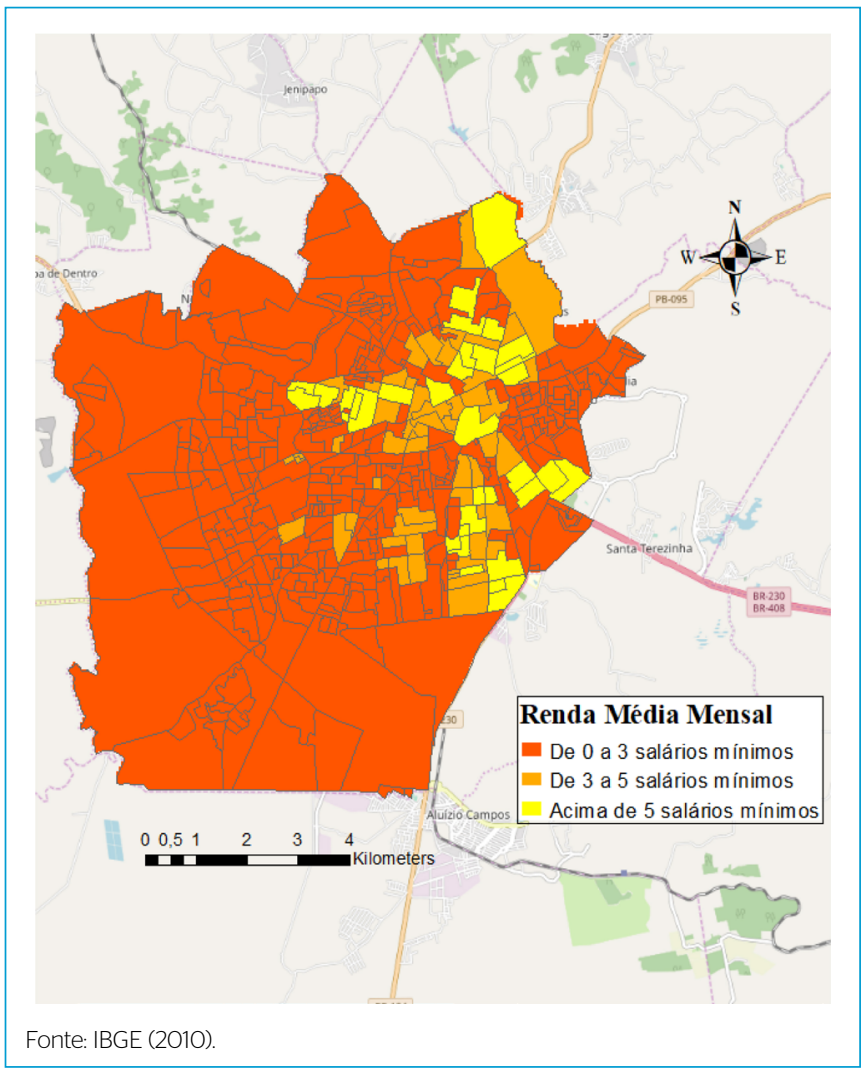

Figura 4 - Renda mensal domiciliar na área urbana de Campina Grande.

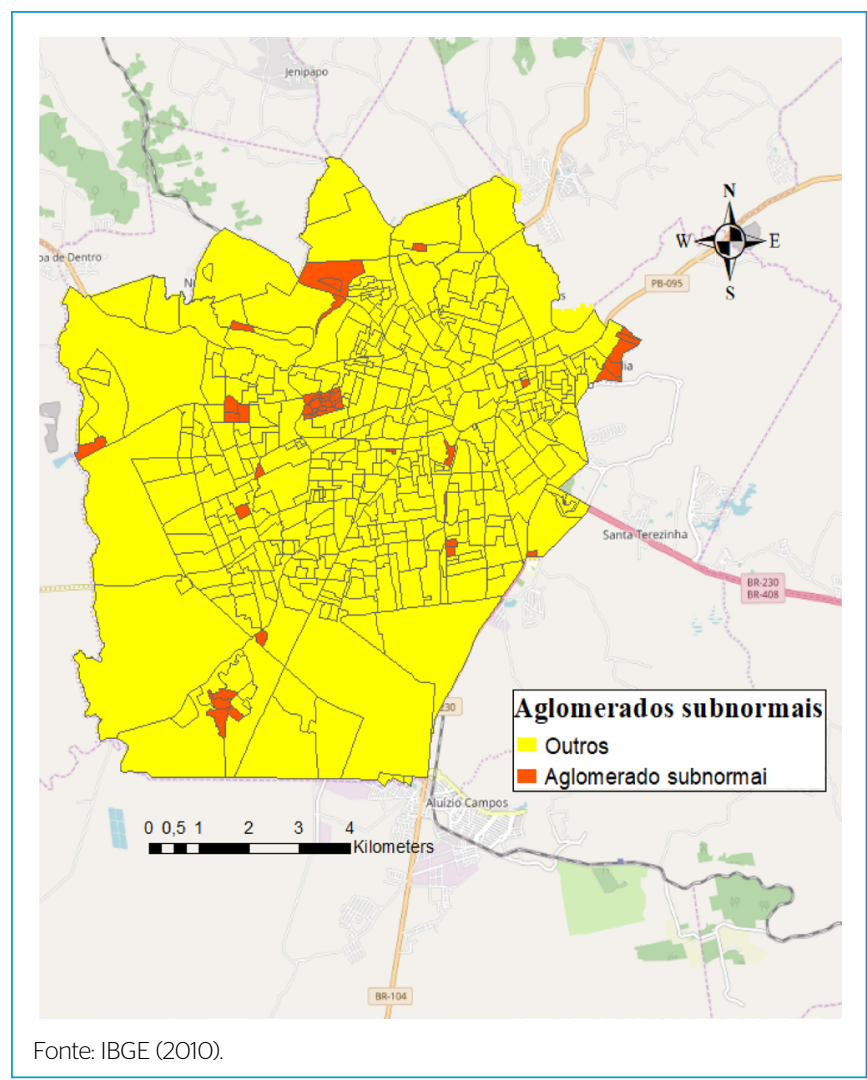

Figura 5 - Presença de aglomerados subnormais na área urbana em Campina Grande.

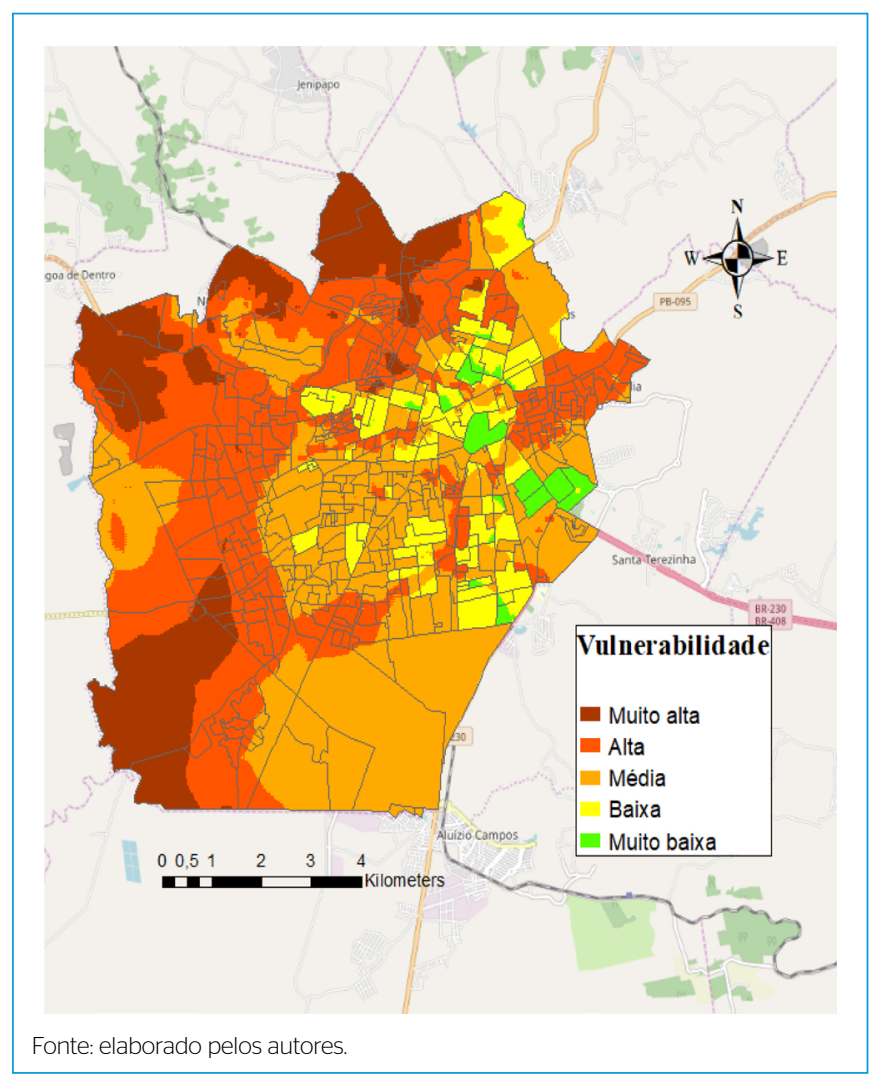

Figura 6 - Mapa de vulnerabilidade para a área urbana de Campina Grande. 


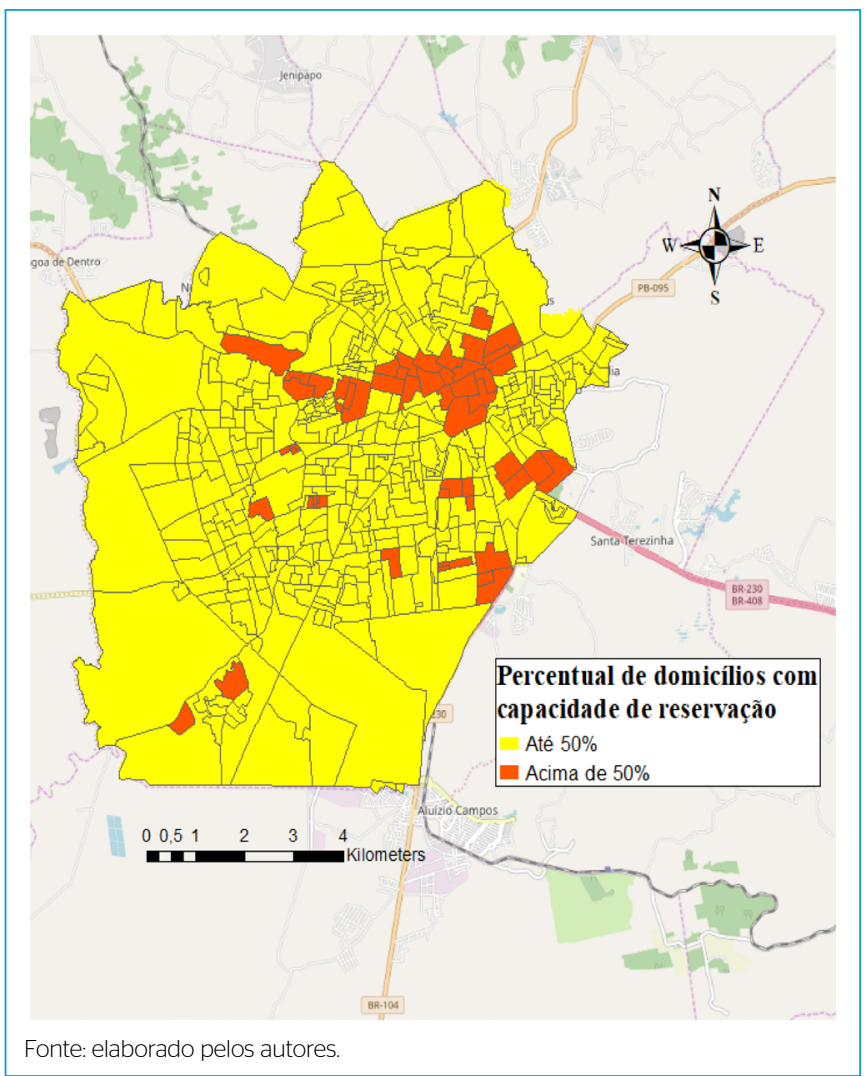

Figura 7 - Domicilios com capacidade de reservação na área urbana de Campina Grande.

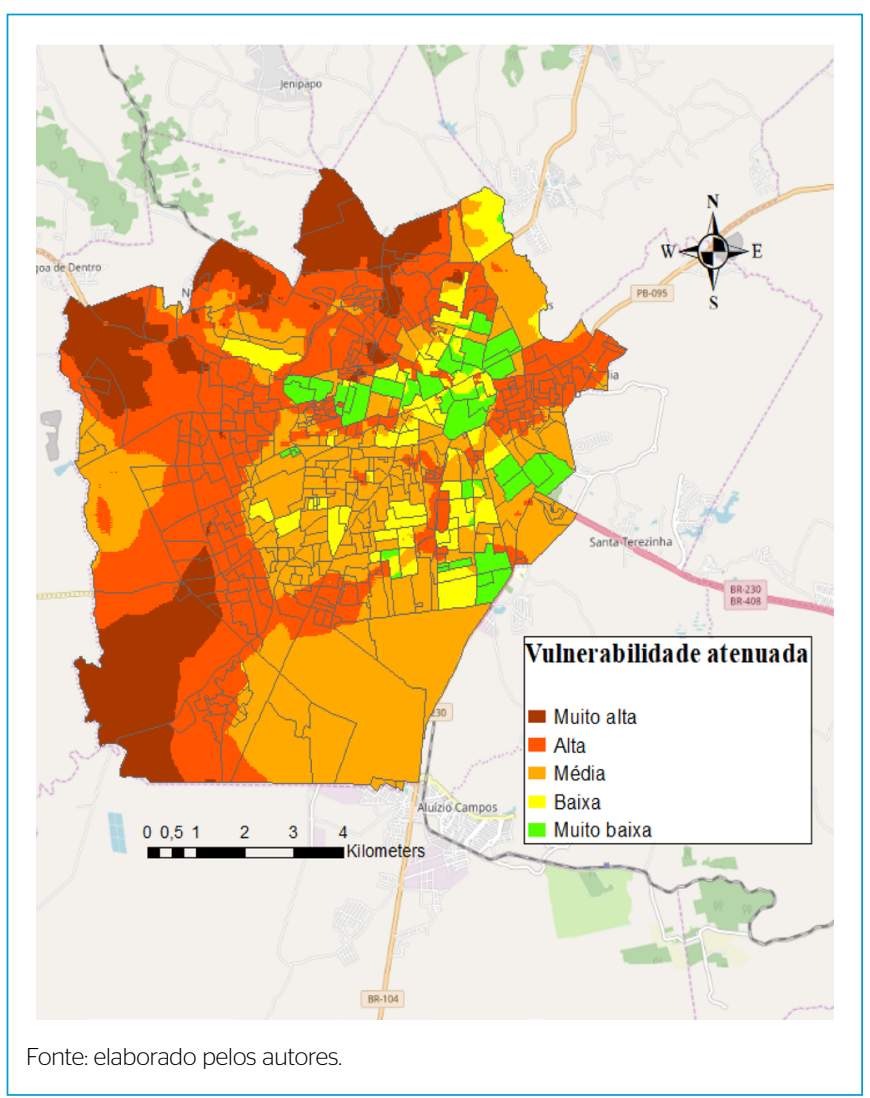

Figura 8 - Mapa de vulnerabilidade atenuada para a área urbana de Campina Grande.
No presente estudo, considerou-se a classe inferior de vulnerabilidade como sendo muito baixa, pelo fato de a intermitência no abastecimento de água ter sido condição permanente em Campina Grande nos últimos 10 anos. Os quatro resultados Sem vulnerabilidade (17\%) do estudo de Grande et al. (2014) foram comparados aos resultados de vulnerabilidade deste estudo, e as classes resultantes foram: muito baixa, baixa e média, consistentes com o risco hidráulico alto e médio dessas localizações, isto é, em áreas cujo risco hidráulico é alto ou médio, a vulnerabilidade se apresenta em diferentes graus, do menor grau (vulnerabilidade muito baixa) até o grau médio (vulnerabilidade média). Os indicadores renda mensal domiciliar e presença de aglomerados subnormais não tornaram a vulnerabilidade mais severa, apontando para o maior peso que o indicador risco hidráulico pode ter sobre a vulnerabilidade nesses quatro resultados. Os oito resultados de vulnerabilidade muito alta e alta restantes (35\%) do estudo de Grande et al. (2014) corresponderam a resultados de vulnerabilidade alta, média e baixa no presente estudo, menos severos, apontando para ponderação do indicador risco hidráulico, o qual nestes casos era médio ou baixo sobre a vulnerabilidade.

Considerando-se a influência que o indicador risco hidráulico mostrou ter sobre os resultados de vulnerabilidade para 12 domicílios da amostra citados acima, passou-se a verificar sua influência nos 11 domicílios cujos resultados de vulnerabilidade foram os mesmos em ambos os estudos. Para as áreas de localização desses 11 domicílios, a vulnerabilidade resultou mais severa do que a classe nominal do risco hidráulico, isto é, para áreas cujo risco hidráulico é alto a vulnerabilidade resultou muito alta, para áreas cujo risco hidráulico é médio a vulnerabilidade resultou alta, e para áreas cujo risco hidráulico é baixo a vulnerabilidade resultou alta, indicando, desta vez, o peso que os indicadores risco hidráulico e Capacidade de reservação têm sobre a vulnerabilidade, a qual resultou mais severa e não foi atenuada em nenhuma dessas localizações, devido ao percentual de domicílios com capacidade de reservação nos respectivos setores censitários ser inferior ou igual a 50\%.

Ainda, verificou-se a coerência dos resultados de vulnerabilidade deste estudo por intermédio de uma análise qualitativa dos dados de capacidade de reservação e renda mensal dos domicílios do estudo de Grande (2016). Em 70\% da amostra de Grande (2016), o resultado de vulnerabilidade é coerente com a capacidade de reservação e a renda mensal; em $22 \%$, o resultado de vulnerabilidade não é coerente com a capacidade de reservação e a renda mensal; e em 9\%, o resultado de vulnerabilidade é aceitável e requer outros critérios para sua confirmação.

Uma resposta política para as situações identificadas de vulnerabilidade ao desabastecimento de água seria a implementação de medidas de gestão de recursos hídricos que reduzam a exposição física em situações de intermitência no abastecimento. Alguns dos indicadores não podem ser modificados, como a renda média mensal e a presença de aglomerados subnormais, portanto não é possível reduzir a sensibilidade dos setores à intermitência. Por outro lado, é possível propor medidas para redução da vulnerabilidade, intervindo no risco hidráulico, por meio da análise do seu papel sobre a vulnerabilidade em cada setor censitário.

A discussão acima indica que a capacidade de reservação é a chave para redução das desigualdades no acesso à água por intermédio da rede de distribuição na cidade. Na falta de adequada cobertura de reservatórios de distribuição na rede, a população tentou reduzir sua vulnerabilidade por meio da implementação de reservação individual nos próprios domicílios. A redução do risco hidráulico e da consequente vulnerabilidade deve, entretanto, ser realizada com intervenções na própria rede de distribuição, para correção da intermitência do serviço. O mapa da Figura 9 localiza as áreas propícias de 
Tabela 1 - Análise comparativa da avaliação da vulnerabilidade entre o presente estudo e o de Grande et al. (2014).

\begin{tabular}{|c|c|c|c|c|c|c|}
\hline Domicilio & $\begin{array}{l}\text { Risco hidráulico de } \\
\text { desabastecimento }\end{array}$ & $\begin{array}{c}\text { Vulnerabilidade } \\
\text { segundo Grande et al. } \\
\text { (2014) }\end{array}$ & $\begin{array}{l}\text { Vulnerabilidade final } \\
\text { (atenuada) segundo } \\
\text { Grande et al. (2014) }\end{array}$ & $\begin{array}{l}\text { Vulnerabilidade } \\
\text { segundo o presente } \\
\text { estudo }\end{array}$ & $\begin{array}{l}\text { Vulnerabilidade } \\
\text { atenuada segundo o } \\
\text { presente estudo }\end{array}$ & Coerência \\
\hline 6 & Alto & Sem & Sem & Média & Média & $\operatorname{sim}$ \\
\hline 5 & Médio & Alta & Alta & Média & Média & $\operatorname{sim}$ \\
\hline 13 & Alto & Muito alta & Muito alta & Muito alta & Muito alta & $\operatorname{sim}$ \\
\hline 10 & Alto & Muito alta & Muito alta & Muito alta & Muito alta & $\operatorname{sim}$ \\
\hline $7 a / 7 b$ & Médio/médio & Muito alta/muito alta & Muito alta/muito alta & Alta/alta & Alta/alta & $\operatorname{sim}$ \\
\hline 2 & Médio & Alta & Alta & Alta & Alta & $\operatorname{sim}$ \\
\hline 8 & Médio & Alta & Alta & Média & Baixa & $\operatorname{sim}$ \\
\hline 12 & Baixo & Alta & Alta & Média & Média & não \\
\hline 14 & Médio & Muito alta & Muito alta & Alta & Alta & $\operatorname{sim}$ \\
\hline 9 & Baixo & Alta & Alta & Baixa & Baixa & $\operatorname{sim}$ \\
\hline 3 & Médio & Alta & Sem & Baixa & Muito baixa & $\operatorname{sim}$ \\
\hline 4 & Baixo & Alta & Alta & Média & Média & $\operatorname{sim}$ \\
\hline 15 & Médio & Alta & Alta & Alta & Alta & $\operatorname{sim}$ \\
\hline 17 & Baixo & Alta & Alta & Média & Média & $\operatorname{sim}$ \\
\hline $1 \mathrm{a} / 1 \mathrm{~b} / 1 \mathrm{c}$ & Alto/alto/médio & Muito alta/muito alta/alta & Muito alta/muito alta/alta & Muito alta/muito alta/alta & Muito alta/muito alta/alta & não \\
\hline 16 & Médio & Alta & Alta & Alta & Alta & $\operatorname{sim}$ \\
\hline 11 & Médio & Alta & Alta & Alta & Alta & $\operatorname{sim}$ \\
\hline 18 & Alto & Muito alta & Muito alta & Muito alta & Muito alta & não \\
\hline 19 & Baixo & Alta & Alta & Alta & Alta & não \\
\hline 20 & Alto & Sem & Sem & Média & Média & $\operatorname{sim}$ \\
\hline 21 & Médio & Alta & Alta & Alta & Alta & não \\
\hline 22 & Alto & Sem & Sem & Baixa & Muito baixa & $\operatorname{sim}$ \\
\hline 23 & Baixo & Alta & Alta & Alta & Média & $\operatorname{sim}$ \\
\hline
\end{tabular}

Fonte: elaborado pelos autores.

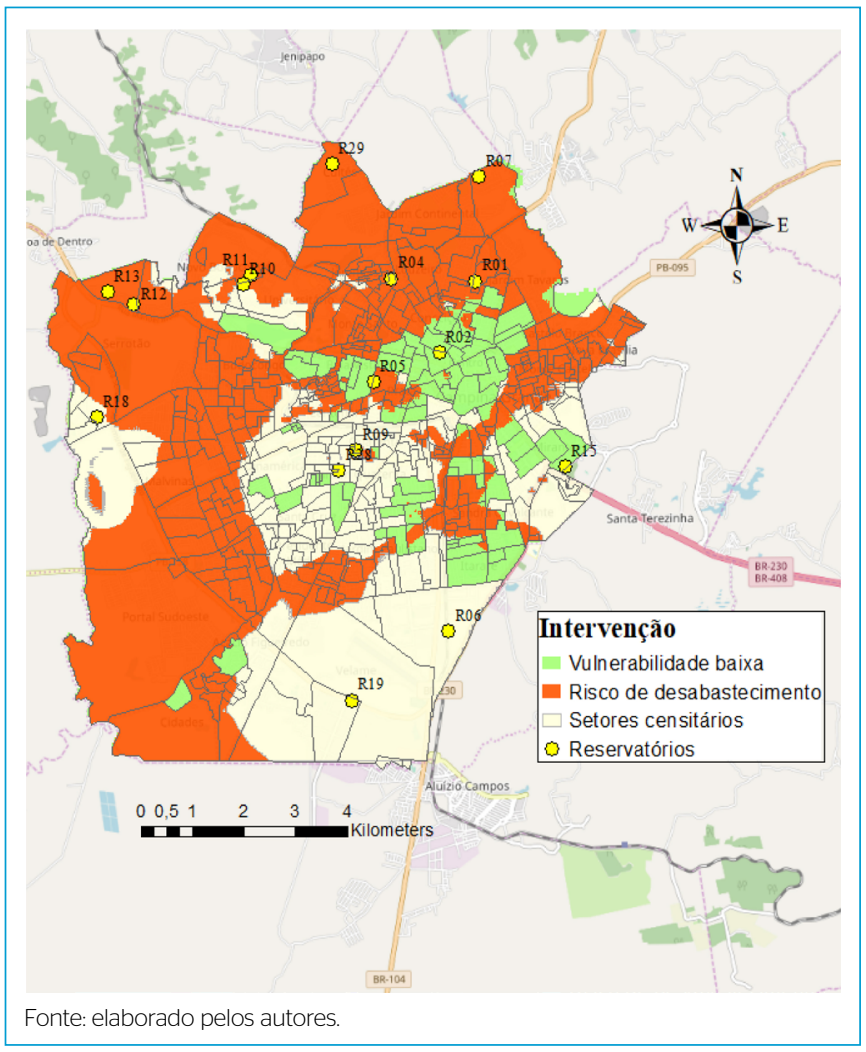

Figura 9 - Localização das possíveis intervenções para redução da vulnerabilidade na área urbana de Campina Grande. intervenção no risco hidráulico, assim como os reservatórios de distribuição de água existentes na área urbana do município. Novos reservatórios de distribuição devem ser implantados de forma a propiciar serviço adequado aos setores censitários cuja vulnerabilidade resultou muito alta, alta e média. Os reservatórios de distribuição de água ficam, atualmente, localizados mais afastados dessas áreas ou em pontos topograficamente desfavoráveis (CORDÃO; RUFINO; ARAÚJO, 2013).

\section{CONCLUSÕES}

O presente estudo expandiu o de Grande et al. (2014), com o aumento do número de classes de vulnerabilidade, bem como o refinamento dos dados referentes à capacidade de reservação domiciliar. Os resultados obtidos demonstram que a vulnerabilidade ao desabastecimento de água ao qual uma cidade está exposta, em situação de intermitência no serviço de abastecimento, pode ser efetivamente caracterizada usando-se dados acessíveis e informações disponíveis da concessionária de abastecimento de água, de imagens de satélite e de dados censitários.

O indicador risco hidráulico determina a vulnerabilidade ao desabastecimento decorrente da intermitência do serviço de distribuição de água. Os indicadores renda média mensal e a presença de aglomerados subnormais agregam informações acerca das condições socioeconômicas da população que interferem na vulnerabilidade ao desabastecimento, permitindo a identificação de áreas vulneráveis, apesar do risco hidráulico baixo, pelas precárias condições de ocupação urbana e renda insuficiente para implantar medidas de adaptação 
no domicílio que reduzam sua vulnerabilidade. O indicador capacidade de reservação informa sobre a capacidade já existente em escala domiciliar para a população se adaptar à intermitência no abastecimento. Apesar de ser uma medida adotada individualmente por cada domicílio em particular e, portanto, não endereçar a causa do desabastecimento, pode reduzir a vulnerabilidade do próprio domicílio.

Para o caso de Campina Grande, a maior parte de sua área urbana está vulnerável às consequências da intermitência do serviço de abastecimento de água. As classes de vulnerabilidade que devem ser foco das ações de intervenção na rede, para minimizar ou eliminar a intermitência no serviço de abastecimento e suas consequências são as muito alta, alta e média. A principal medida deve ser a implantação de novos reservatórios de distribuição, localizados de modo a reduzir os valores do indicador risco hidráulico.

\section{AGRADECIMENTOS}

Os autores agradecem à Secretaria de Planejamento da Prefeitura Municipal de Campina Grande, pela cessão de dados. Este artigo é um produto da Rede Clima, dos Projetos BRAMAR, Secas e Cheias e INCTs Observatório das Metrópoles e Mudanças Climáticas. Agradecem também a contribuição do editor e dos dois revisores anônimos, que trouxe mais qualidade ao trabalho.

\section{CONTRIBUIÇÕES DOS AUTORES}

Diniz, T.G.: Conceituação, Curadoria de Dados, Análise Formal, Escrita — Primeira Redação, Escrita - Revisão e Edição. Del Grande, M.H.: Conceituação, Análise Formal, Escrita - Primeira Redação, Escrita - Revisão e Edição. Galvão, C.O.: Conceituação, Análise Formal, Escrita — Primeira Redação, Escrita — Revisão e Edição.

\section{REFERÊNCIAS}

AGATHOKLEOUS, A.; CHRISTODOULOU, S. Vulnerability of Urban Water Distribution Networks under Intermittent Water Supply Operations. Water Resources Management, v. 30, n. 13, p. 4731-4750, 2016.

ALVES, H.P.F. População e Desmatamento no Vale do Ribeira: integração de dados censitários com dados de sensoriamento remoto dentro da estrutura de um sistema de informação geográfica (GIS). Focus, n. Xiii, p. $1-25,2000$.

BORUFF, B.; BIGGS, E.; PAULI, N.; CALLOW, N.; CLIFTON, J. Changing water system vulnerability in Western Australia's Wheatbelt region. Applied Geography, v. 91, p. 131-143, 2018. https://doi.org/10.1016/j.apgeog.2017.12.016

CORDÃO, M.J.S.; RUFINO, I.A.A.; ARAÚJO, E.L. Geotecnologias aplicadas ao planejamento de sistemas de abastecimento de água urbanos: uma proposta metodológica. Engenharia Sanitária e Ambiental, v. 18, n. 3, p. 263274, 2013. https://doi.org/10.1590/S1413-41522013000300009

CUTTER, S.L.; BORUFF, B.J.; SHIRLEY, W.L. Social vulnerability to environmental hazards. Social Science Quarterly, v. 84, n. 2, p. 242-261, 2003. https://doi.org/10.1111/1540-6237.8402002

GRANDE, M.H. Distribuição e acesso à água em Campina Grande: uma análise a partir da Ecologia Política. Tese (Doutorado em Recursos Naturais) - Universidade Federal de Campina Grande, Campina Grande, 2016.

GRANDE, M.H.; GALVÃO, C.; MIRANDA, L.; GUERRA SOBRINHO, L.D. A percepção dos usuários sobre os impactos do racionamento de água em suas rotinas domiciliares. Ambiente \& Sociedade, v. 19, n. 1, p. 163-182, 2016. https://doi.org/10.1590/1809-4422asoc150155r1v1912016

GRANDE, M.H.; GALVÃO, C; MIRANDA, L.; RUFINO, I. Environmental equity as a criterion for water management. Proceedings of the International Association of Hydrological Sciences, v. 364, p. 519-525, 2014. https://doi. org/10.5194/piahs-364-519-2014
ILAYA-AYZA, A.E.; BENITEZ, J.; IZQUIERDO, J.; PÉREZ-GARCÍA, R. Multi-criteria optimization of supply schedules in intermittent water supply systems. Journal of Computational and Applied Mathematics, v. 309, p. 695-703, 2017. https://doi.org/10.1016/j.cam.2016.05.009

INSTITUTO BRASILEIRO DE GEOGRAFIA E ESTATISTICA (IBGE). Censo 2010. IBGE, 2010. Disponível em: https://censo2010.bge.gov.br/. Acesso em: 16 out. 2018

MALTA, F.S.; COSTA, E.M.; MAGRINI, A. Índice de vulnerabilidade socioambiental: uma proposta metodológica utilizando o caso do Rio de Janeiro, Brasil. Ciência \& Saúde Coletiva, v. 22, n. 12, p. 3933-3944, 2017. https://doi.org/10.1590/1413-812320172212.25032017

MUKESH, S.B.; KOMAL, C.; ALEXANDER, K. Land Use/Cover and Vulnerability Mapping Through Remote Sensing and GIS In Astrakhan, Russia. Journal of Earth Science \& Climatic Change, v. 8, n. 1, 2017.

RÊGO, J.C.; GALVÃO, C.O.; ALBUQUERQUE, J.P.T.; RIBEIRO, M.M.R.; NUNES, T.H.C. Gestão de Recursos Hídricos e a Transposição de Águas do Rio São Francisco para o Açude Epitácio Pessoa - Boqueirão. In: SIMPÓSIO BRASILEIRO DE RECURSOS HÍDRICOS, 22., 2017. Anais [...]. 2017.

RUNFOLA, D.M.; RATICK, S.; BLUE, J.; MACHADO, E.A.; HIREMATH, N.: GINER, N.; WHITE, K.; ARNOLD, J. A multi-criteria geographic information systems approach for the measurement of vulnerability to climate change. Mitigation and Adaptation Strategies for Global Change, v. 22, n. 3, p. 349 368, 2017. https://doi.org/10.1007/s11027-015-9674-8

SECRETARIA DE PLANEJAMENTO (SEPLAN); PREFEITURA DE CAMPINA GRANDE (PMCG). Mapa digital de Campina Grande. Campina Grande: SEPLAN/PMCG, 2014.

SIMUKONDA, K.; FARMANI, R.; BUTLER, D. Intermittent water supply systems: causal factors, problems and solution options. Urban Water Journal, v. 15, n. 5. p. 488-500, 2018. https://doi.org/10.1080/1573062X.2018.1483522 